\title{
Case Study of Misoscale Convective Echo Behavior Associated with Cumulonimbus Development Observed by Ka-band Doppler Radar in the Kanto Region, Japan
}

\author{
Namiko Sakurai, Koyuru Iwanami, Takeshi Maesaka, Shin-ichi Suzuki, \\ Shingo Shimizu, Ryohei Misumi, Dong-soon Kim, and Masayuki Maki \\ National Research Institute for Earth Science and Disaster Prevention (NIED), Tsukuba, Japan
}

\begin{abstract}
Simultaneous observations of cumulonimbi by a Ka-band Doppler radar $(\mathrm{KaDR})$ and an X-band polarimetric Doppler radar (MP-X) were performed during the summer of 2011 in the Kanto region, Japan to study the process of cumulonimbus initiation and development. A cumulonimbus developed up to $12 \mathrm{~km}$ above sea level (ASL) in the mountainous western part of the Kanto region on the morning of 18 August 2011, and its initiation and development were observed by the two radars. A misoscale convective echo which was newly detected in an RHI or PPI scan and developed vertically (RHI scan) or spatially (PPI scan) was labeled as a 'new misoscale convective echo' (NMCE). In the developing stage (DS), NMCEs occurred one after another, and the echo top height and maximum reflectivity of each individual echo gradually increased. In the first half of the DS, the NMCEs appeared between 2 and $5 \mathrm{~km}$ ASL. In contrast, in the second half of the DS, the NMCEs' appearance height stepped up to between 5 and $12 \mathrm{~km}$ ASL. These results suggest that the ascent of NMCE appearance height is one of the key factors in the prediction of deep convection, which later causes localized heavy rainfall.
\end{abstract}

\section{Introduction}

Localized heavy rainfall events, one type of local severe weather, often occur in the summer season in the Tokyo Metropolitan Area, Japan. The localized heavy rainfall resulting from the sudden development of an isolated thunderstorm often causes damage in the form of flash floods and traffic congestion. At some locations in the Tokyo Metropolitan Area, rain water falling at 50 $\mathrm{mm} \mathrm{hr}{ }^{-1}$ cannot flow into the local sewage systems within short periods of time (Bureau of Urban Development Tokyo Metropolitan Government 2007). To mitigate the disasters suffered from the localized heavy rainfall, it is necessary to develop a technique for the early detection of cumulonimbi that will cause localized heavy rainfall and for the high accuracy prediction of localized heavy rainfall.

Previous studies have investigated the generation of precipitation system such as the height of first radar echo appearance with centimeter-wavelength radar (e.g., Braham 1958; Kobayashi and Inatomi 2003). The centimeter-wavelength radar is appropriate for precipitation observation but has insufficient sensitivity to observe a cloud particle. Because a cloud particle is much smaller than a rain drop, higher sensitivity and higher spatial resolution than those of the centimeter-wavelength radar is necessary to observe the fine structure of the cumulonimbus in the initiation and early developing stage (DS).

Millimeter-wavelength radar is a useful tool for observing the initiation and early DS of cumulonimbi because it has higher sensitivity and higher spatial resolution than those of conventional weather radars (S-, C-, and X-band radars; centimeter-wavelength

Corresponding author: Namiko Sakurai, National Research Institute for Earth Science and Disaster Prevention, 3-1 Tennodai, Tsukuba, Ibaraki 305-0006, Japan. E-mail: sakurain@bosai.go.jp. (C2012, the Meteorological Society of Japan. radars). However, there is only one previous study of the initiation and development of a cumulonimbus using a vertically-pointing millimeter-wavelength radar (W-band with $3 \mathrm{~mm}$ wavelength) in the Kanto region of Japan (Kobayashi et al. 2011). The National Research Institute for Earth Science and Disaster Prevention (NIED) of Japan set up a Ka-band Doppler radar (KaDR) at Hidaka City, Saitama Prefecture, Japan, and was then able to carry out simultaneous observations of cumulonimbi using the KaDR and an existing X-band polarimetric Doppler radar (MP-X) located at Ebina City (Fig. 1). The KaDR's observation area has a high frequency of thunderstorm initiation during the summer season, when compared with the whole Kanto region. This area was investigated using JMA radar data and an AMeDAS (Automated Meteorological Data Acquisition System) radar precipitation map designed by Horie and Tomine (1998). On the morning of 18 August 2011, a cumulonimbus developed within the observation range of the KaDR, and we succeeded in observing the cloud from initiation to the DS using the KaDR and from the DS to the dissipation stage using the MP-X. The objective of this study is to describe the process of initiation and development of the cumulonimbus observed on 18 August 2011 by using the KaDR and MP-X data. We investigate the time variation of characteristics of the cumulonimbus such as echo top height and echo intensity associated with the cumulonimbus development using the KaDR data primarily.

\section{Observation and data description}

The NIED KaDR, which is a track-mounted Ka-band Doppler radar (Iwanami et al. 2001), was installed at Hidaka (HDK) in Saitama Prefecture, Japan at the end of July 2011 (Fig. 1). The period of intensive observation by the KaDR was 1 to 26 August 2011. The KaDR operated at a frequency of $35.35 \mathrm{GHz}$, had a beam width of $0.28^{\circ}$, a pulse width of $0.5 \mu \mathrm{s}$, a Nyquist velocity of $8.5 \mathrm{~m} \mathrm{~s}^{-1}$, and an observation range of $30 \mathrm{~km}$ (Fig. 1). Nearly the entire observation area for the KaDR was contained within the observation area for the MP-X (Fig. 1). The sensitivity of the KaDR's reflectivity signal was $-23 \mathrm{dBZ}$ at $10-\mathrm{km}$ range. In this study, reflectivity data are used for analysis, after applying the attenuation correction determined from the relationship between reflectivity factor and specific attenuation calculated by Iwanami et al. (2005).

The KaDR's observation method was as follows. To detect the initiation of a cumulonimbus, sector plan position indicator (PPI) scans at three elevation angles $\left(7.3^{\circ}, 10.5^{\circ}\right.$ and $\left.14.9^{\circ}\right)$ were conducted. The three angles were chosen for the efficient detection of a cumulonimbus initiation between 2 and $4 \mathrm{~km}$ above sea level (ASL) and between 10 and $20 \mathrm{~km}$ in range. Kobayashi and Inatomi (2003) used X-band radar data and reported that the first radar echo of a summer thundercloud often occurs between 2 and $4 \mathrm{~km}$ ASL in the southern Kanto region, including the observation area of the KaDR. Once a radar echo was detected, the scan strategy was changed: the sector PPI scans were stopped and the range height indicator (RHI) scans toward the detected echo were started, although a sector PPI scan was inserted after every two or four RHI scans to obtain the position of the radar echo. This scan strategy, combining RHI and sector PPI scans, was continued until 


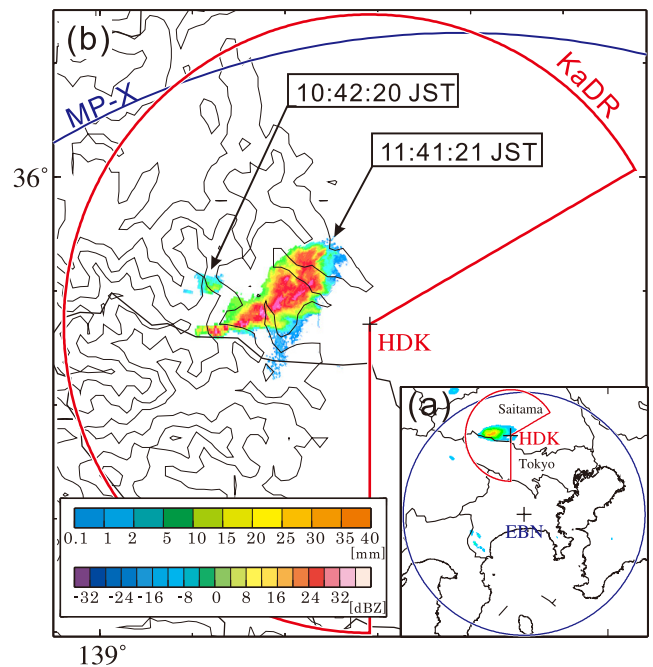

Fig. 1. A box at the lower-right corner (a) shows locations of a Ka-band Doppler radar (KaDR) installed at Hidaka City (cross at HDK) and an $\mathrm{X}$-band polarimetric Doppler radar (MP-X) installed at Ebina City (cross at $\mathrm{EBN})$. Observation ranges were $30 \mathrm{~km}(\mathrm{KaDR})$ and $83 \mathrm{~km}(\mathrm{MP}-\mathrm{X})$ (circles). Rainfall amount from 10:30 to 12:30 JST on 18 August 2011 is shaded. Elevation contours are shown at every $300 \mathrm{~m}$ in (b) and reflectivity at an elevation of $0.7^{\circ}$ at 10:42:20 and 11:41:21 JST on 18 August 2011 are shaded.

the radar echo decayed and dissipated or the radar echo left the observation area of the KaDR.

The MP-X performed sector scans to collect data from the cumulonimbus after its radar echo was detected by the KaDR. The sector scans had a $60^{\circ}$ azimuthal width and 17 elevation angles from $0.7^{\circ}$ to $15.6^{\circ}$. The time resolution of the sector volume scan was 2 minutes and the observation range of the MP-X was $83 \mathrm{~km}$ (Fig. 1). The MP-X has a pulse width of $0.5 \mu$ s and a beam width of $1.3^{\circ}$. The MP-X's reflectivity $\left(Z_{H}\right)$ data are corrected for rainfall attenuation, using the relationship between specific attenuation and specific differential phase $\left(K_{D P}\right)$ derived from differential propagation phase $\left(\Phi_{D P}\right)$ data (Maesaka et al. 2011), and used for analyses in this study.

\section{Results}

Figures 2 and 3 show a surface weather map and sounding profiles at Tateno, respectively, at 09:00 Japan Standard Time (JST $=$ UTC +9 hours) on 18 August 2011. On that day, there was a stationary front in the northern part of Japan, but no synoptic disturbances in the Kanto region, and warm and humid air moved into the Kanto region near the surface. Relative humidity between the surface and $5.5 \mathrm{~km}$ ASL was relatively high, but was low above $5.5 \mathrm{~km}$ ASL (Fig. 3a). A westerly wind was dominant from the surface to $13 \mathrm{~km}$ ASL (Fig. 3b). Supplement 1 shows an animation of the sector PPI scans of reflectivity observed by the KaDR from 10:32:13 to 12:37:36 JST on 18 August 2011. A radar echo was first detected at a height of about $4 \mathrm{~km}$ ASL at 10:32:13 JST. The following radar echoes occurred one after another in the same part of the mountainous area due to an updraft induced by local thermal circulation and the slope effect for about 30 minutes. Each radar echo gradually developed and migrated eastward. New echoes generated west side of old echoes and they became a mass of radar echo with multicell structure. The reflectivity and the dimensions of the radar echoes became large and the radar echo descended the slope of the mountain (Fig. 1b and Supplement 1). The maximum reflectivity reached $60.2 \mathrm{dBZ}$, as observed by the MP-X at 11:50:21 JST (figure not shown). The radar echo passed over the KaDR site at around 12:15 JST, left the observation area, and then decayed. The MP-X observed the last radar echo

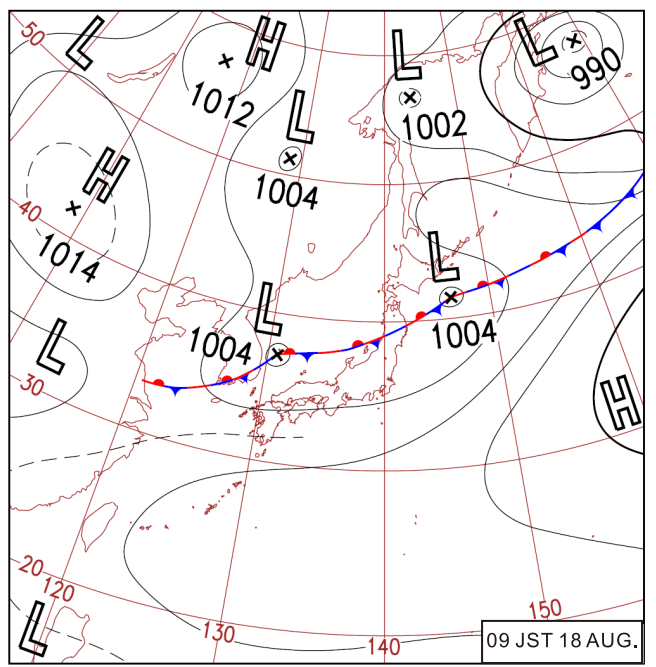

Fig. 2. Surface weather map at 09:00 JST on 18 August 2011.
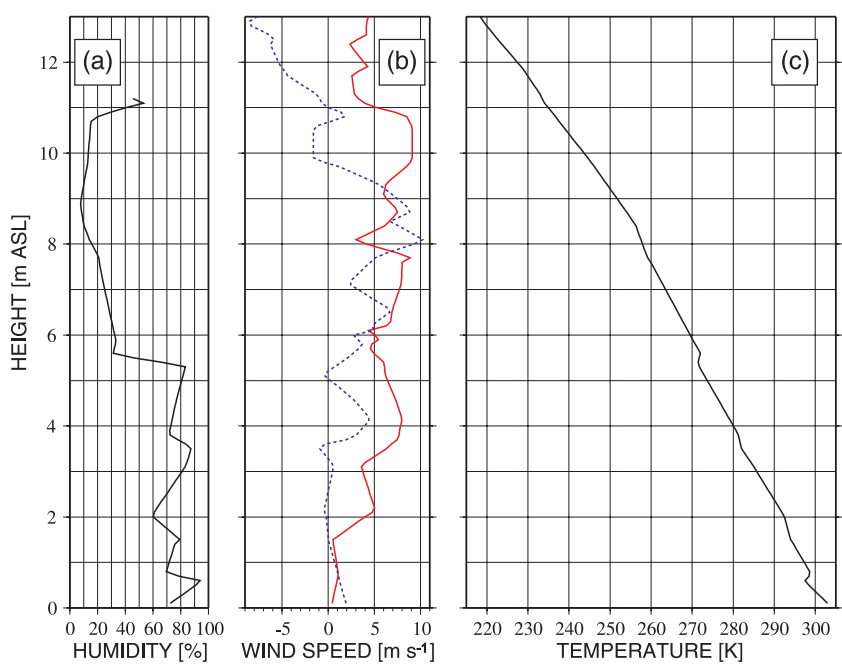

Fig. 3. Sounding profiles of relative humidity (a), horizontal wind (b), and temperature (c) observed at Tateno at 09:00 JST on 18 August 2011. Solid red line (dashed blue line) in (b) indicates zonal wind (meridional wind).

at around 12:24 JST (figure not shown). Figure 1a shows the total rainfall amount calculated by using the data of the MP-X and X-band polarimetric Doppler radars of the Ministry of Land, Infrastructure, Transport and Tourism (MLIT) between 10:30 and 12:30 JST, and the maximum rainfall amount was $29 \mathrm{~mm}$, observed about $12 \mathrm{~km}$ west of the HDK site.

The evolution of the cumulonimbus is investigated by using the time series of its echo top height and maximum reflectivity observed by the KaDR during the DS from 10:36:56 to 11:59:56 JST, and the DS was separated into three steps (Fig. 4). In the beginning of the DS, convective activity is considered shallow because the echo top height was from 2.0 to at most $5.5 \mathrm{~km} \mathrm{ASL}$. The maximum reflectivity was about $0 \mathrm{dBZ}$ on average and increased slowly. In the middle of the DS, the maximum echo top height of each identified echo reached from 4.5 to at most $7.0 \mathrm{~km}$ ASL. The maximum reflectivity increased sharply and had a peak at $30 \mathrm{dBZ}$. In the end of the DS, the echo top height exceeded $7.5 \mathrm{~km}$ ASL with a maximum attained height of $12 \mathrm{~km}$ ASL. The maximum reflectivity was about $35 \mathrm{dBZ}$ on average and changed little. This is because dynamic range of the KaDR was insufficient. These increases in echo top height and maximum reflectivity correspond to the increase in the diameter and/or the number of precipitation 

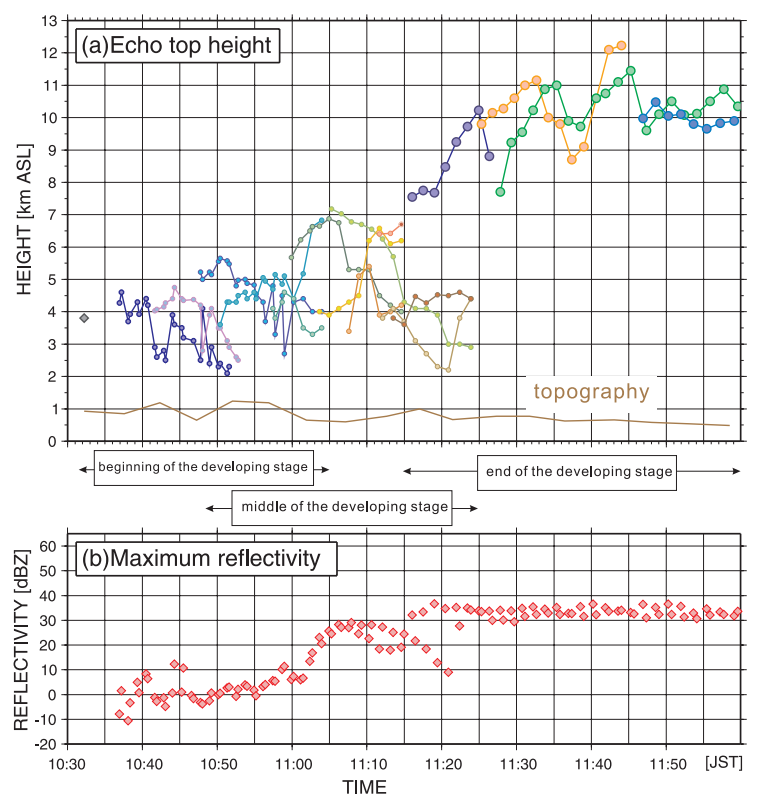

Fig. 4. Time series of echo top height (a) and maximum reflectivity (b) of a cumulonimbus observed by the KaDR in the DS on 18 August 2011. The echo top height values for each identified echo are connected by the same colored line in (a). The rhomboid mark in (a) indicates the height of first echo detected by the KaDR.

particles within the cumulonimbus which developed vertically.

The time lag between the first detection of radar echo by the $\mathrm{KaDR}$ and the MP-X is also investigated, because the KaDR has higher sensitivity than the MP-X and the KaDR can detect a smaller precipitation particle and/or the smaller number of the precipitation particles than the MP-X. In previous studies (e.g., Braham 1958; Kobayashi and Inatomi 2003), the first detected echo of a cumulonimbus by radar is called the first echo. Using this definition, the first echo time lag was $25 \mathrm{~min} 24 \mathrm{~s}$ in our case: the KaDR and MP-X first detected an echo of the cumulonimbus at 10:32:13 and 10:57:37 JST, respectively. The radar echo was observed about 20 (60) km from the KaDR (MP-X). The minimum detectable intensity of the KaDR (MP-X) was $-16.4 \mathrm{dBZ}$ (about $20 \mathrm{~km})(11.9 \mathrm{dBZ}$ (about $60 \mathrm{~km})$ ).

Utilizing high sensitivity and high spatial resolution of the $\mathrm{KaDR}$, we focused on the fine structure and development process of the cumulonimbus. Three representative examples of the fine structure and development process of the cumulonimbus are shown in Fig. 5. In the case of the first example, a misoscale convective echo appeared to the side of a pre-existing echo (Fig. 5a) and developed vertically (Figs. 5b and 5c). It did not merge with the pre-existing echo. In the case of the second example, a misoscale convective echo appeared to the side of a pre-existing echo (Fig. 5d), merged with the pre-existing echo, and the pre-existing echo developed vertically (Figs. $5 \mathrm{e}^{-}-5 \mathrm{~g}$ ). In the case of the third example, a misoscale convective echo appeared to the side of a pre-existing echo like a branch and developed vertically: the upper part of the pre-existing echo was replaced by the developed misoscale convective echo (Figs. 5h-5m). We label a misoscale convective echo which was newly detected in an RHI or PPI scan and developed vertically (RHI scan) or spatially (PPI scan) such as the echoes in Figs. 5a, 5d, and 5h as a "new misoscale convective echo' (NMCE). The height of each NMCE in Fig. 6 indicates the middle height between top and bottom for each NMCE. The NMCEs appeared in two height regions in the DS. In the first half of the DS (including the beginning and first half of the middle of the DS), the NMCEs were observed between 2 and $5 \mathrm{~km} \mathrm{ASL}$. In the second half of the DS (including the second half of the middle and the end of the DS), the NMCEs were observed between 5 and $12 \mathrm{~km}$ ASL. The NMCE height was low during shallow
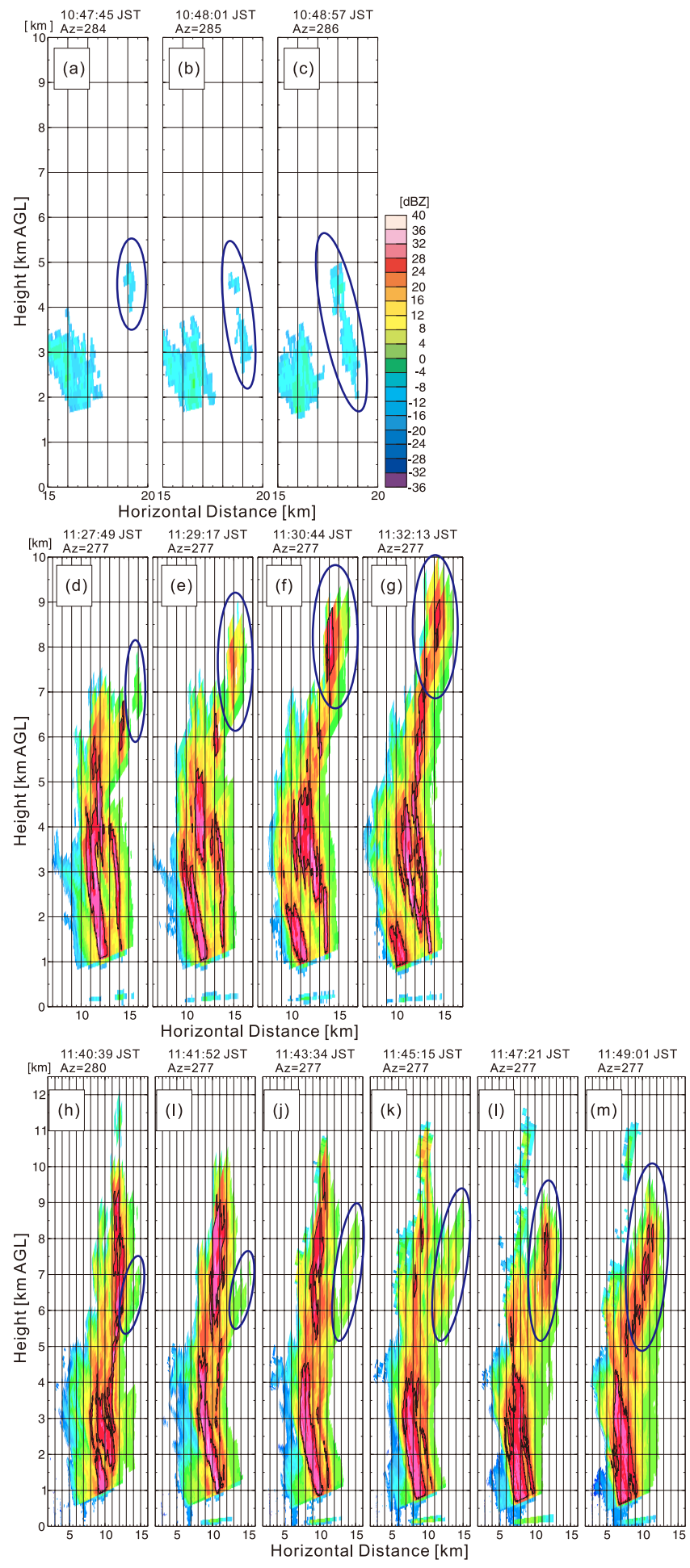

Fig. 5. Vertical cross-sections of reflectivity observed by KaDR RHI scanning on 18 August 2011. The blue ovals indicate positions of the NMCE and the following echoes.

convection and then jumped in the second half of the DS. Thus, we consider that the height of the NMCEs is useful information to judge whether a cloud will be deep convection or not later.

Furthermore the time lag is estimated for each NMCE by comparing sector PPI data observed by the KaDR and the MP-X. The time lag tended to be smaller as convection developed: the lags were 8 min $59 \mathrm{~s}$ and $7 \mathrm{~min} 59 \mathrm{~s}$ in the middle of the DS, compared with $2 \mathrm{~min} 28 \mathrm{~s}$ in the end of the DS, which suggested that 


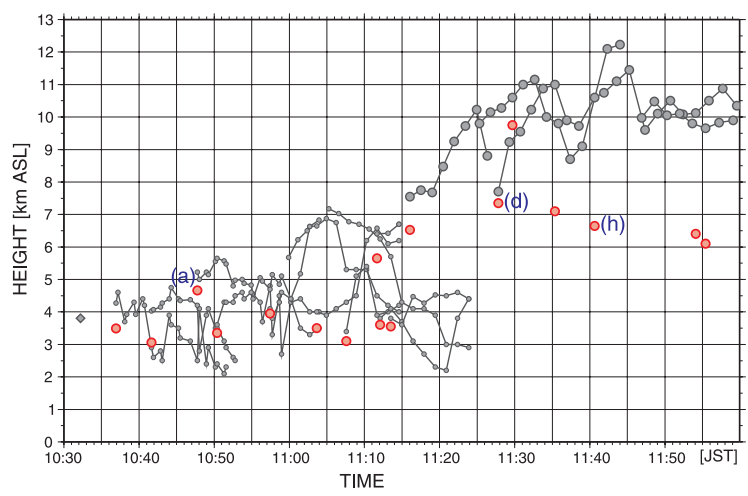

Fig. 6. Time series of the NMCE height (red) and echo top height (gray) of a cumulonimbus observed by the KaDR in the DS on 18 August 2011. The echo top height is the same as that in Fig. 4a. The rhomboid mark indicates the height of first echo detected by the KaDR. NMCEs labeled as (a), (d), and (h) correspond to the NMCEs in Figs. 5a, 5d, and 5h.

the growth speed of precipitation particles and/or increasing speed of the number of precipitation particles became fast associated with the cumulonimbus development.

The relationship between stable layers in the atmosphere and the cumulonimbus development process is investigated. Figure $3 \mathrm{c}$ shows a temperature profile (T) observed at Tateno at 09:00 JST on 18 August 2011. There were stable layers at around $0.8,1.8$, $3.8,5.5$, and $8.1 \mathrm{~km}$ ASL. The $\Delta T / \Delta z$ of each layer was smaller than the moist adiabatic lapse rate (Ohno 2001). The two layers at around 0.8 and $5.5 \mathrm{~km} \mathrm{ASL}$ were temperature inversion layers and are extremely stable. It is well known that the height of convective activity relates to that of the inversion layer (Nodzu et al. 2006). In the beginning of the DS, development of echo top height was suppressed by the stable layers at around 3.8 and $5.5 \mathrm{~km}$ ASL (Fig. 4a). The following convective activity broke through these stable layers in the middle of the DS, and echo top height finally reached $12 \mathrm{~km}$ ASL in the end of the DS. Similarly, we investigated a relationship between the height of a NMCE and that of a stable layer. In the first half of the DS, the NMCE height was concentrated between 2 and $5 \mathrm{~km}$ ASL, with most NMCE heights located just below $4 \mathrm{~km} \mathrm{ASL}$, where a stable layer existed. In the second half of the DS, the NMCE height was concentrated between 5 and $8 \mathrm{~km}$ ASL, where stable layers existed. Thus, it is clear that echo top height and NMCE height corresponded well to the height of stable layers in the DS. However, the reason why the NMCE height is related to that of a stable layer is unknown. To clarify the mechanism of NMCE appearance, an accumulation of case studies and an investigation of the cloud microphysics process (using a numerical model such as the bin microphysics model) are needed.

\section{Summary}

Intensive observation of cumulonimbi with a Ka-band Doppler radar $(\mathrm{KaDR})$ and an X-band polarimetric Doppler radar (MP-X) was conducted in the western Kanto region, Japan in August 2011. Using the KaDR, with its high sensitivity and high spatial resolution, we could observe the initiation and development of a cumulonimbus on 18 August 2011. On the morning of this day, a cumulonimbus emerged in the mountainous western part of the Kanto region, and at the location of maximum rainfall delivered a total of $29 \mathrm{~mm}$. From the analysis of the KaDR and MP-X data, three characteristics of the initiation and development process of the cumulonimbus were described in this paper.

1. The echo top height and maximum reflectivity developed in three steps. In the beginning of the DS, convection was shallow and reflectivity was weak. The MP-X could not detect any cumulonimbus echo in the beginning of the DS. In the middle of the DS, the echo top height and reflectivity increased, and the MP-X first detected an echo. In the end of the DS, echo top height and reflectivity became higher than those in the middle of the DS. The maximum echo top height exceeded $12 \mathrm{~km}$ ASL in the end of the DS.

2. The time lag between the detection of NMCEs by the KaDR and the MP-X became smaller as the cumulonimbus developed.

3. The NMCE was concentrated in two height regions. In the first half of the DS, the NMCE was observed between 2 and $5 \mathrm{~km}$ ASL; however, the NMCE was observed between 5 and $12 \mathrm{~km}$ ASL in the second half of the DS.

By focusing on the fine structure in a cumulonimbus such as the NMCE, we propose to utilize the variation of NMCE height to identify a cloud that would grow through deep convection such as a cumulonimbus. We showed the effectiveness of the KaDR for the early detection of a cumulonimbus. Because we presented only a single case study, it is necessary to perform additional case studies in order to obtain a comprehensive understanding of the process of cumulonimbus development.

\section{Acknowledgements}

We are grateful for the constructive comments of anonymous reviewers and the editor (Dr. H. Yamada) that helped to improve the paper. Radar data provided by MLIT were used for the calculation of rainfall amount due to the cumulonimbus observed on 18 August 2011 (Fig. 1a).

\section{Supplement}

Animation of Ka-band radar echo patterns of sector PPI scans from 10:32:13 to 12:37:36 JST on 18 August 2011.

\section{References}

Braham Jr., R., 1958: Cumulus cloud precipitation as revealed by radar -Arizona 1955. J. Meteor., 15, 75-83.

Horie, H., and K. Tomine, 1998: A study of generation and movement of air mass thunderstorms over Kanto area in summer 1995. Tenki, 45, 441-453 (in Japanese).

Iwanami, K., R. Misumi, M. Maki, T. Wakayama, K. Hata, and S. Watanabe, 2001: Development of a multiparameter radar system on mobile platform. Proc. 30th Conf. on Radar Meteor, 104-106.

Iwanami, K., Y. Chono, T. Harimaya, J. Testud, M. Maki, R. Misumi, and S.-G. Park, 2005: Retrieval of vertical rain rate profile by dual-frequency radar data. Proc. 32th Conf. on Radar Meteor., 104-106.

Kobayashi, F., and N. Inatomi, 2003: First radar echo formation of summer thunderclouds in southern Kanto, Japan. J. Atmos. Electricity, 23, 9-19.

Kobayashi, F., T. Takano, and T. Takemura, 2011: Isolated cumulonimbus initiation observed by $95-\mathrm{GHz}$ FM-CW radar, $\mathrm{X}$-band radar, and photogrammetry in the Kanto region, Japan. SOLA, 7, 125-128.

Maesaka, T., M. Maki, K. Iwanami, S. Tsuchiya, K. Kieda, and A. Hoshi, 2011: Operational rainfall estimation by X-band MP radar network in MLIT, Japan. Proc. 35th Conf. on Radar Meteor, $12-18$.

Nodzu, M., S. Ogino, Y. Tachibana, and M. D. Yamanaka, 2006: Climatological description of seasonal variations in lower-tropospheric temperature inversion layers over the Indochina peninsula. J. Climate, 19, 3307-3319.

Ohno, H., 2001: Thunderstorm and meso scale meteorology. Tokyodo Publishing Co. Ltd., 309 pp (in Japanese).

Manuscript received 15 June 2012, accepted 19 August 2012

SOLA: http://www.jstage.jst.go.jp/browse/sola 